\title{
A Joint Link Budget and MAC/PHY Cross-Layer Model for DiffServ IEEE 802.11e WLANs
}

\author{
Roger Pierre Fabris Hoefel
}

\begin{abstract}
An analytical medium access control (MAC) and physical (PHY) cross-layer model to calculate the link budget and to estimate the cell coverage of IEEE 802.11e wireless local area networks (WLANs) is derived. A comparison between numerical and simulation results is carried out assuming the IEEE 802.11a and IEEE 802.11g PHY layers.
\end{abstract}

Index Terms - 802.11e, 802.11a, 802.11g, link budget, coverage.

\section{INTRODUCTION}

The release of the first IEEE 802.11 standard, that specifies the MAC and the original 1 and 2 Mbps frequency-hopping (FH) and direct sequence (DS) PHY layers in 1997 paved the way for a world wide development of a standardized cost-effective scalable technology for WLANs. The synergy between a cost-effective scalable technology and a huge commercial success has been driving intensive research activities in order to provide quality-of-service (QoS) in WLANS, resulting in the evolving IEEE 802.11e standard.

In 1997, B. P. Crow et al [1] published one of the first papers to qualitatively explain the IEEE 802.11 protocol, with particular emphasis on the medium access control, (MAC) layer. In 2000, Bianchi [2] proposed a seminate analytical bi-dimensional Markov model to estimate the performance of IEEE 802.11 networks operating under saturated traffic conditions over ideal channels. In 2002, Qiao et al [3] derived an analytical model that takes the non-ideal channel into account on the performance of IEEE 802.11a WLANs. However, they assumed a simplistic model for the MAC layer. In 2006, we carried out a unified theoretical analyses upon the performance of IEEE 802.11a WLANs over uncorrelated and correlated fading channels when the basic access (BA) and request-to-send/clear-tosend (RTS/CTS) MAC protocols are operating simultaneously [4]. In 2004, J. W. Robison and T.S. Randhawa [5] presented one of the first comprehensive analytical models to assess the performance of IEEE 802.11 WLANs with QoS schemes (i.e the IEEE 802.11e standard). However, they assumed an ideal channel. In 2006, we proposed an analytical model to estimate the goodput (net throughput) and delay of IEEE 802.11e MAC and PHY protocols operating under a realistic radio channel environment [6].

On the other hand, the estimation of the cell coverage is a fundamental issue to a fast and cost effective deployment of WLANs in enterprise environments. Therefore, researchers from academy, developers of equipments and broadband wireless integrators have published peer-review papers, application notes, white papers and software tools in order to systematize and optimize the cell planning procedures. The Wireless Connections (www.wirelessconnections.net) and Terabean (www.terabeam.com), integrators of wireless

Manuscript received Abril 17, 2007.

Roger Pierre Fabris Hoefel is with Department of Electrical Engineering, Federal University of Rio Grande do Sul (UFRGS), Porto Alegre, Rio Grande do Sul, Brazil; E-mail: roger.hoefel@ufrgs.br. broadband solutions, have provided basic software tools to estimate the link budget, In 2002, researchers from AT\&T Labs have presented in [7] a basic link analyzes for IEEE 802.11 outdoor cellular networks. In 2006, Ramela and de Rezende [8] proposed an analytical model to estimate the reduction of interference, and consequently to allow coverage or capacity gains, with the use of directional antennas in ad hoc networks. In [9], we proposed an analytical model to estimate the cell coverage of IEEE 802.11a, 800.11b and 802.11g networks.

To the best of our knowledge, there is still room in the open literature for analytical works that take into account the channel load, goodput, channel modelling, receiver structures and link analyses for IEEE 802.11e networks in an integrated way. To accomplish our goals, this paper is organized as follows. Section II summarizes our analytical model that has been developed to assess the goodput of IEEE EDCF 802.11e protocol under non-ideal channel assumptions. Section III and IV describe the IEEE 802.11a and 802.11g PHY layers, respectively. A generic methodology to calculate the link budget to IEEE 802.11 WLANs is developed in Section V. Section VI presents a comparative performance assessment of goodput and cell coverage of IEEE 802.11e WLANs over flat fading Rayleigh channels. Section VII shows the final remarks.

\section{IEEE 802.11e EDCF}

The enhanced distributed coordination function (EDCF) protocol provides QoS in IEEE 802.11 distributed coordination function (DCF) networks by establishing four access classes (ACs). This ACs are parameterized by the following parameters: (1) the arbitration interframe spacing for the $j$ th $A C$ : $A I F S(j)$; (2) the minimum contention window (CW) size for the jth AC: $C W_{\min }(j)$; (3) the maximum $C W$ size for the $j$ th $A C$ : $C W_{\max }(j)$; (4) the maximum number of retransmission attempts for the $j$ th $A C: m(j)$.

To transmit a MAC protocol data unit (MPDU) a QoS station (QSTA) must defer its transmission until the channel is idle for a time period equal to the $A I F S(j)$ :

$$
\operatorname{AIFS}(A C)=\operatorname{AIFSN}(A C) \cdot \sigma+\operatorname{SIFS} \text {, }
$$

where $\operatorname{AISFN}(A C)$ is an integer number for each AC QSTA, $\sigma$ is the slot time, and SIFS is the short interframe spacing.

Henceforth, we shall summarize a modeling that we have developed to estimate the following variables: (1) the probability $\tau(j)$ (i.e. the transmission probabilities for the $j$ th AC QSTA); (2) the conditional probability $p(j)$ (i.e. the probability that the $j$ th AC QSTA collides considering that a transmission was carried out) for the IEEE 802.11e EDCF protocol. We have assumed that:

$$
\text { 1) there is a fixed number of } n \text { QSTAs, } n=\sum_{j=1}^{2} n(j) \text {, where } n(j)
$$

denotes the number of the QSTAs that belong to the $j$ th AC. So, 
our analytical model considers two ACs of QSTAs $\left(N_{c}=2\right)$, as well as postulated in [5] using a different analytical approach;

2) the QSTAs operate in saturation conditions, i.e. each QSTA has a MPDU to transmit after the completion of each successful transmission;

3) the MPDUs transmitted by the QSTAs that belong to the $j$ th AC collide with a constant and independent conditional collision probability $p(j)$;

4) the window size at backoff stage $i$ is $W_{i}(j)=2^{i} W(j)$, where $W(j)$ is the MAC CW size parameter, $C W_{\min }(j)$, for the $Q S T A(j)$;

5) the maximum window size for the $Q S T A(j)$ is denoted as $W_{m}(j)=$ $C W_{\max }(j)-1=2^{m(j)} W(j)-1$;

6) the internal collisions in the AC queues of the QSTAs and post backoff procedure are not taken into account

When a QSTA that belongs to the lowest AC transmit a frame, then the conditional probability that this frame collides can be estimated by

$p(1)=1-\left\{(1-\tau(1))^{n(1)-1} \cdot(1-\tau(2))^{n(2)}\right\}$,

i.e. QSTAs that belong to the first and second ACs can collide among them during all the vulnerable collision period.

Considering that a given QSTA that belongs to the highest priority AC transmit a frame, then the conditional probability that this QSTA collides can be stated as

$p(2)=1-\left[\begin{array}{l}q_{\text {zone }=A}(1-\tau(2))^{n(2)-1}+ \\ q_{\text {zone }=B}(1-\tau(2))^{n(2)-1} \cdot(1-\tau(2))^{n(1)}\end{array}\right]$.

Notice that in (3), due to the deterministic IFS priority, there are two distinct contention zones: (1) zone $=A$ where only the QSTAs that belong to the highest class can collide among them; (2) zone $=B$ where the highest priority QSTA can collide with the other $n(2)-1$ QSTAs of the same AC and with the $n(1)$ QSTAs that belong to the lower priority AC. The probability that a collision occurs in the zone A can be approximated as

$q_{\text {zone }=A} \approx \frac{\operatorname{AIFS}(1)-\operatorname{AIFS}(2)}{\operatorname{AIFS}(1)}$,

i.e. the relative priority due to the AIFS of AC2 QSTAs in relation to the AIFS of AC1 QSTAs. Naturally, the probability that a collision takes place in the zone $B$ can be approximated by (5) when there are only two different ACs.

$q_{\text {zone }=B}=1-q_{\text {zone }}=A$.

When $\operatorname{AIFS}(1)=\operatorname{AIFS}(2)$, we have that

$p(2)=1-\left[(1-\tau(2))^{n(2)-1} \cdot(1-\tau(1))^{n(1)}\right]$,

since $q_{\text {zone }=A}=0$ and $q_{\text {zone=B }}=1$. Therefore, (2) and (6) can also be used to model the conditional collision probabilities system when the priorities are only established by the different parameters of the CW.

Using a procedure similar to that one developed in [4] and [6], we can show that the probability that a QSTA that belongs to the lowest AC transmits in a randomly slot time is given by

$\tau(1)=q_{\text {zone }=B} \cdot \frac{b_{0,0}(1)}{[1-p(1)] \cdot S(1)}$.

We denote as $b_{0,0}(1)$ the probability that a $Q S T A(j)$ be in the time slot 0 at the backoff stage 0 (see 10 below) and

$S(j)=S_{r t s}(j) \cdot S_{c t s}(j) \cdot S_{d}(j) \cdot S_{a c k}(j)$,

is the probability that the frames transmitted in the RTS/CTS atomic cycle are not corrupted due to noise and interference. $S_{r t s}(j), S_{c t s}(j), \quad S_{d}(j)$ and $S_{a c k}(j)$ denote, respectively, the probability that the RTS, CTS, MPDU and acknowledgment (ACK) frames be transmitted with success by $Q S T A(j)$ in the case of a collision has not happened.

The probability that a QSTA of lower priority transmits in a randomly chosen slot time is given by [6]

$$
\tau(2)=\frac{b_{0,0}(2)}{[1-p(j)] \cdot S(j)} \text {. }
$$

Comparing (7) and (9), we can see that the constant $q_{\text {zone=B }}$ in (7) models the relative effect of deterministic IFS priority on the transmission probability of lower priority QSTAs.

Finally, the probability that a $Q S T A(j)$ be in the time slot 0 at the backoff stage 0 is given by (10), where the subscript $j$ was dropped to avoid a cumbersome notation [2].

$$
b_{0,0}(j)=\frac{2 \cdot(1-p) \cdot S \cdot[1+2 \cdot S \cdot(p-1)]}{1+2^{m} W \cdot[1+(-1+p) \cdot S]^{m}+(-1+p) \cdot S \cdot\left\{2+W+2^{m} W \cdot[1+(-1+p) \cdot S]^{m}\right.} \cdot
$$

Assuming only two different ACs, then four non-linear equations are necessary to determine the stationary system state:

1. Eq. (7) that gives $\tau(1)$ when parameterized by AC1 parameters using (10) with $j=1$;

2. Eq. (2) that models $p(1)$;

3. Eq. (9) that gives $\tau(2)$ when parameterized by AC2 parameters using (10) with $j=2$;

4. Eq. (3) that estimates the conditional collision probability for AC2 QSTAS.

\section{A. Goodput}

The goodput (or net throughput) in bits per second (bps) can be modeled as the ratio of the MPDU payload bits transmitted with success to the average cycle time, i.e.

$G_{b p s}=\sum_{j=1}^{N_{C}} G_{b p s}(j)=\frac{\bar{N}_{r t s}(j)}{\bar{T}_{r t s}(j)+\bar{I}}$,

where the goodput for the QSTAs that belong to the $j$ th AC is denoted by $G_{b p s}(j)$.

The average number of payload bits transmitted with success for RTS/CTS MAC scheme is given by

$\bar{N}_{r t s}(j)=8 \cdot N_{p l}(j) \cdot P_{s}(j) \cdot P_{t r}(j) \cdot S_{r t s}(j) \cdot S_{c t s}(j) \cdot S_{m p}(j) \cdot S_{a c k}(\mathrm{j}) \cdot$

Assume again that $N_{c}=2$. Hence, the probability that there is no collision on the channel conditioned to the fact that at least one QSTA that belongs to the lower QoS class transmit is given by

$$
\begin{aligned}
P_{S}(1) & =\frac{q_{\text {zone }=B} \cdot\left[n(1) \cdot \tau(1) \cdot(1-\tau(1))^{n(1)-1} \cdot(1-\tau(2))^{n(2)}\right]}{P_{\text {tr }}(1)} \\
& =\frac{q_{\text {zone }}=B \cdot\left[n(1) \cdot \tau(1) \cdot(1-\tau(1))^{n(1)-1} \cdot(1-\tau(2))^{n(2)}\right]}{q_{\text {zone }}=B \cdot\left[1-\cdot(1-\tau(1))^{n(1)}\right]}, \\
& =\frac{n(1) \cdot \tau(1) \cdot(1-\tau(1))^{n(1)-1} \cdot(1-\tau(2))^{n(2)}}{1-(1-\tau(1))^{n(1)}}
\end{aligned}
$$

where $P_{t r}(1)$ is the probability that there is at least one transmission in the considered slot time located in zone $B$ (i.e. the only zone where the AC1 QSTAs are allowed to transmit).

Correspondingly, the probability that there is no collision on the channel conditioned to the fact that at least one 
QSTA that belongs to the higher class transmits is given by

$P_{s}(2)=\left\{\begin{array}{l}\frac{q_{\text {zone }=A} \cdot\left[n(2) \cdot \tau(2) \cdot(1-\tau(2))^{n(2)-1}\right]}{P_{t r}(2)}+ \\ \frac{q_{\text {zone }=B} \cdot\left[n(2) \cdot \tau(2) \cdot(1-\tau(2))^{n(2)-1} \cdot(1-\tau(1))^{n(1)}\right]}{P_{t r}(2)}\end{array}\right\}$.

Using (4-5), the probability that a QSTA that belongs to the AC2 transmit is given by (15), where it was used (5). We reinforce again that the AC2 QSTAs are allowed to transmit in both zones $A$ and $B$, whereas the AC1 QSTA can only transmit in zone A.

$$
\begin{aligned}
P_{t r}(2) & =q_{\text {zone }=A} \cdot\left[1-(1-\tau(2))^{n(2)}\right]+q_{\text {zone }=B} \cdot\left[1-(1-\tau(2))^{n(2)}\right] . \\
& =1-(1-\tau(2))^{n(2)}
\end{aligned}
$$

The mean cycle time for RTS/CTS schemes is given by .

$\bar{T}_{r t s}(j)=\bar{B}_{s, r t s}(j)+\bar{B}_{f 1, r t s}(j)+\bar{B}_{f 2, r t s}(j),+\bar{B}_{f 3, r t s}(j)+B_{f 4, r t s}(j)+B_{f 5, r t s}(j)$,

The average busy time when the transmission is successful using the RTS/CTS scheme is given by

$\bar{B}_{S}(j)=P_{S}(j) \cdot P_{t r}(j) \cdot S_{r t s}(j) \cdot S_{c t s}(j) \cdot S_{m p}(j) \cdot S_{m a c}(j) \cdot\left[\operatorname{AIFS}(j)+T_{r t s}\left(m_{r t s}\right)+\right.$ $\left.a+\operatorname{SIFS}+T_{c t s}\left(m_{c t s}\right)+a+\operatorname{SIFS}+T_{m p}\left(m_{m p}\right)+a+\operatorname{SIFS}+T_{a c k}\left(m_{a c k}\right)+a\right]$

where where $a$ is the propagation delay. $T_{r t s}\left(m_{r t s}\right), T_{c t s}\left(m_{c t s}\right)$ and $T_{a c k}\left(m_{a c k}\right)$ denote the time necessary to transmit the RTS, CTS and ACK control frames when it is used the PHY mode $m_{r t s}, m_{c t s}$ and $m_{a c k}$, respectively. $T_{m p}\left(m_{m p}\right)$ is the period of time necessary to transmit a MPDU when it is used the PHY mode $m_{m p}$ [4].

The waste time occurs due to collisions of RTS control frames, as given by

$$
\bar{B}_{f 1, r t s}(j)=P_{t r}(j) \cdot\left(1-P_{S}(j)\right) \cdot\left(\operatorname{AIFS}(j)+T_{r t s}\left(m_{r t s}\right)+a\right) .
$$

$$
\bar{B}_{f 2, r t s}(j), \quad \bar{B}_{f 3, r t s}(j), \quad \bar{B}_{f 4, r t s}(j) \text { and } \bar{B}_{f 5, r t s}(j)
$$

model the average time that the channel is busy with unsuccessful transmissions, due to noise and interference, of RTS, CTS, data and ACK frames, respectively. Finally, the average time that a slot time is idle is given by (23).

$$
\begin{aligned}
& \bar{B}_{f 2, r t s}(j)=P_{t r}(j) \cdot P_{s}(j) \cdot\left(1-S_{r t s}(j)\right) \cdot\left[\operatorname{AIFS}(j)+T_{r t s}\left(m_{r t s}\right)+a\right] \\
& \bar{B}_{f 3, r t s}(j)=P_{t r}(j) \cdot P_{S}(j) \cdot S_{r t s}(j) \cdot\left(1-S_{c t s}(j)\right) \cdot\left[\begin{array}{l}
\operatorname{AIFS}(j)+T_{r t s}\left(m_{r t s}\right)+a+ \\
\operatorname{SIFS}+T_{c t s}\left(m_{c t s}\right)+a
\end{array}\right] .
\end{aligned}
$$

$\bar{B}_{f 4, r t s}=P_{t r}(j) \cdot P_{s}(j) \cdot S_{r t s}(j) \cdot S_{c t s}(j) \cdot\left(1-S_{m p}(j)\right)[\operatorname{AIFS}(j)+$

$$
\left.T_{r t s}\left(m_{r t s}\right)+a+\operatorname{SIFS}+T_{c t s}\left(m_{c t s}\right)+a+\operatorname{SIFS}+T_{m p}\left(m_{m p}\right)+a\right] .
$$

$\bar{B}_{f 5, r t s}(j)=P_{t r}(j) \cdot P_{S}(j) \cdot S_{r t s}(j) \cdot S_{m p}(j) \cdot\left(1-S_{a c k}(j)\right)[\operatorname{AIFS}(j)+$

$T_{r t s}\left(m_{r t s}\right)+a+\operatorname{SIFS}+T_{c t s}\left(m_{c t s}\right)+a+\operatorname{SIFS}+$

$\left.T_{m p}\left(m_{m p}\right)+a+\operatorname{SIFS}+T_{a c k}\left(m_{a c k}\right)+a\right]$.

$\overline{\mathrm{I}}=\sum_{\mathrm{j}=1}^{\mathrm{N}_{\mathrm{c}}}(1-\operatorname{Ptr}(\mathrm{j})) \cdot \sigma$.

\section{IEEE 802.11 PHY LAYER}

The IEEE 802.11a [10] is based on Orthogonal Frequency Division Multiplexing (OFDM) using a total of
52 subcarriers, of which 48 subcarriers carry actual data and four subcarriers are pilots used to facilitate coherent detection. The OFDM symbol interval, tSymbol, is set to $4 \mu \mathrm{s}$. Therefore, the channel symbol rate $R_{s}$ is of 12 Msymbols/sec. Tab. 1 shows the OFDM PHY characteristics.

TABLE I

The IEEE 802.11a PHY modes.

\begin{tabular}{|c|c|c|c|c|c|}
\hline Mode $m$ & Mod. & Data Rate & Mode $m$ & Mod. & Data Rate \\
\hline 1 & BPSK & $6 \mathrm{Mbps}$ & 5 & 16-QAM & 24 Mbps \\
\hline 2 & BPSK & $9 \mathrm{Mbps}$ & 6 & 16-QAM & $36 \mathrm{Mbps}$ \\
\hline 3 & QPSK & $12 \mathrm{Mbps}$ & 7 & $64-\mathrm{QAM}$ & $48 \mathrm{Mbps}$ \\
\hline 4 & QPSK & $18 \mathrm{Mbps}$ & 8 & $64-\mathrm{QAM}$ & $54 \mathrm{Mbps}$ \\
\hline
\end{tabular}

Assuming hard decision decoding and a maximum ratio combining (MRC) receiver, then we have shown in [4] a set of analytical expressions to estimate the frame success probabilities (i.e $S_{c t s}, S_{r t s}, S_{m p}$ and $S_{a c k}$ ) for uncorrelated and correlated flat fading Rayleigh channels. It is assumed hard decision Viterbi decoding.

The RTS and CTS control frames must be transmitted at one of the rates of the basic service set (BSS) so that they can be decoded by all the STAs in the same network. The mandatory BSS basic rate set is $\{6$ Mbps, 12 Mbps, 24 Mbps . The ACK control frame must be transmitted using the mandatory BSS basic rate that is less than or equal to the rate of the data frame that it is acknowledging.

\section{IEEE 802.11G EXTENDED RATE PHY LAYER}

This paper is focused on the major mode of the IEEE 802.11g, i.e. the Extended-Rate PHY layer Orthogonal Frequency Division Modulation (ERP-OFDM). Basically, it was carried out minor changes in the 802.11a PHY layer in order to adapt it to the $2.4 \mathrm{GHz}$ band and to allow coexistence with older networks that implement the 802.11b standard.

The ERP-OFDM 802.11g PHY layer uses the same modulation mode of IEEE 802.11a PHY layer, as shown in Tab. 1. However, the RTS and CTS control frames are transmitted using the CCK/DSSS modulation scheme. This approach allows that the 802.11b and 802.11g STAS can coexist in the same area, since $802.11 \mathrm{~b}$ STAs can listen the control frames transmitted by 802.11g STAs and, consequently, set their network allocation vectors (NAVs). Therefore, as shown in Fig. 1, if the transmission of RTS and CTS are successful then the sender STA can transmit its MPDU without interruption of other 802.11b STAs in the same area. Notice that this reservation scheme would be impossible if the RTS and CTS frame were transmitted using OFDM, since the 802.11b STAs could not decode this signalling scheme. The duration of the RTS, CTS and ACK control frames can be calculated as specified in [9].

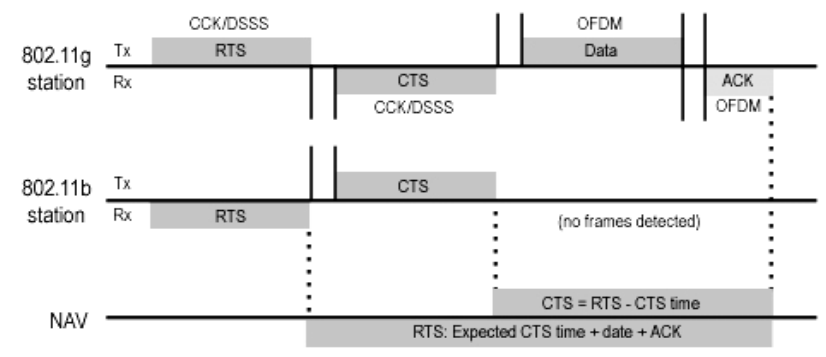

Fig. 1. IEEE 802.11g with RTS/CTS protection mechanism. 
Analytical expressions to calculate the frame success probability for CCK/DSSS modulation scheme can be found in [11].

\section{LINK BUDGET}

The link budget is a balance sheet of power gains and losses. It takes into account the effects of transmission and reception resources, noise and interference sources, signal attenuation and fading.

Tab. 2 shows a link budget for radio channel link access in IEEE 802.11 networks. The detailed explanation of each row entry is shown below Tab. 2 .

TABLE II

Access point to mobile stations link budget

\begin{tabular}{|c|c|c|c|}
\hline Line & Symbol & & Notes: \\
\hline 1 & $\overline{\mathrm{P}_{\mathrm{tx}}}$ & Transmitter Power $(\mathrm{dBm})$ & \\
\hline 2 & $\mathrm{~L}_{\text {conc }}$ & Connectors Loss $(d B)$ & \\
\hline 3 & $\mathrm{~L}_{\text {cable }}$ & Cable Loss $(d B)$ & \\
\hline 4 & $\mathbf{P}_{\text {ir }}$ & $\begin{array}{l}\text { Power of the Intentional } \\
\text { Radiator }(\mathrm{dBm})\end{array}$ & (\#1-\#2-\#3) \\
\hline 5 & $\mathrm{G}_{\mathrm{tx}}$ & $\begin{array}{l}\text { Transmitter Antenna Gain } \\
\text { (dBi) }\end{array}$ & \\
\hline 6 & EIRP & Transmitter EIRP (dBm) & $(\# 4+\# 5)$ \\
\hline 7 & $\mathrm{~L}_{\mathrm{p}}$ & Path Loss $(d B)$ & \\
\hline 8 & $\mathrm{X}_{\sigma}$ & Shadowing Margin $(d B)$ & \\
\hline 9 & $\mathrm{G}_{\mathrm{rx}}$ & Receiver Antenna Gain (dBi) & \\
\hline 10 & $\mathbf{P}_{\mathrm{rx}}$ & Received Power ( $\mathrm{dBm})$ & (\#6-\#7-\#8+\#9) \\
\hline 11 & $\mathrm{R}_{\mathrm{b}}$ & Data Rate (dB-bit/s) & \\
\hline 12 & $\mathbf{E}_{\mathbf{b}}$ & Energy per bit (dB-Joules) & $(\# 10-\# 11)$ \\
\hline 13 & $\mathrm{~N}_{\mathrm{o}}$ & $\begin{array}{l}\text { Noise Spectral Density } \\
(\mathrm{dBm} / \mathrm{Hz})\end{array}$ & \\
\hline 14 & $\mathrm{~W}$ & System Bandwidth dB-Hz & \\
\hline 15 & $\mathrm{~F}$ & Noise Figure $(d B)$ & \\
\hline 16 & $\mathbf{N}$ & Noise Power $(\mathrm{dBm})$ & $(\# 13+\# 14+\# 15)$ \\
\hline 17 & $\overline{M_{I}}$ & Interference Margin $(d B)$ & \\
\hline 18 & $\mathbf{I}$ & $\begin{array}{c}\text { Interference-plus-noise } \\
\text { power }(\mathrm{dBm})\end{array}$ & $(\# 16+17)$ \\
\hline 19 & $(\mathrm{SINR})_{\mathrm{rx}}$ & Received SINR (dB) & \\
\hline 20 & (SINR) $)_{\text {est }}$ & Estimated $\mathrm{E}_{\mathrm{b}} / \mathrm{N}_{\mathrm{o}}(\mathrm{dB})$ & \\
\hline
\end{tabular}

1. The transmitted power is set to attend the following constraints: (a) it must be less than the maximum transmitted power available (e.g. $15 \mathrm{dBm}$ for Orinoco cards and $30 \mathrm{dBm}$ for Proxim cards); (b) the maximum effective isotropic power must attend the maximum values allowed by the regulatory agencies (see item 4).

2. The typical loss in connectors is $0.25 \mathrm{~dB}$ per connector

3. The typical loss range is from $1 \mathrm{~dB} / \mathrm{m}$ to $0.1 \mathrm{~dB} / \mathrm{m}$. For instance, the loss for the following cables are: (a) $100 \mathrm{~dB} / 100 \mathrm{~m}$ for the RG 58; (b) for $12 \mathrm{~dB} / 100 \mathrm{~m}$ for the Heliax $1 / 2$ "; (c) $7 \mathrm{~dB} / 100 \mathrm{~m}$ for Heliax 7/8".

4. The power output of the intentional radiator refers to the power at the end of the last cable or connector before the antenna.

5. Typical antenna gains: (a) $0 \mathrm{dBi}$ for ommidirectional antenna; (b) $2 \mathrm{dBi}$ for a simple integrated antenna; (c) $5 \mathrm{dBi}$ for a simple external antenna.

6. The maximum EIRP is regulated by state agencies, such as Federal Communication Commission (FCC) in the USA and European Telecommunications Standards Institute (ETSI) in European Union. The FCC limits the EIRP as follows: (a) 30 $\mathrm{dBm}$ for $802.11 \mathrm{~b} / \mathrm{g}$ [10, p.257]; (b) $22 \mathrm{dBm}$ (channels 36-48) and $29 \mathrm{dBm}$ (channels 52-64) for 802.11a. On the other hand, the ETSI limits the EIRP by $20 \mathrm{dBm}$ for $802.11 \mathrm{~b} / \mathrm{g}$ [10, p.
257]. The maximum EIRP set by the Brazilian Telecommunications Agency (ANATEL) is given by: (a) 26 $\mathrm{dBm}$ for the $2.4 \mathrm{GHz}$ range; (b) $20 \mathrm{dBm}$ for the bandwidth between 5.15 to $5.35 \mathrm{GHz}$. In this paper, we have assumed the typical values of $24 \mathrm{dBm}$ for $802.11 \mathrm{~b}$ and $802.11 \mathrm{~g}$ and 20 $\mathrm{dBm}$ for $802.11 \mathrm{a}$ since the Wi-Fi products are traded worldwide [10, p. 447]. The EIRP is given by (24). In this paper we have fixed the EIRP, then we must set the power delivery by the 802.11 card using (25).

$E I R P=P_{t x}-L_{\text {conc }}-L_{\text {cable }}+G_{a, t x}$.

$P_{t x}=E I R P+L_{c o n c}+L_{c a b l e}-G_{a, t x}$

7. We have assumed a path loss model based on the breakpoint model. It has the free space loss distance exponent $(n=2)$ for the first 10 meters and a distance exponent of $n=3.5$ when the distance $d$ increases above the breakpoint. Hence, the path loss can be written as

$L_{p}(d)=L_{0}+\left\{\begin{array}{l}20 \log d, \quad 1<d \leq 10 \mathrm{~m} \\ 20+20+35 \log \frac{d}{10}, \quad d>10 \mathrm{~m}\end{array}\right.$,

where $L_{0}$ is the free space path loss at reference distance of $1 \mathrm{~m}$ :

$$
L_{0}=40 \log \frac{4 \pi \cdot f}{c},
$$

where $f$ is the frequency and $c$ is velocity of light [12].

8. The shadowing is modeled by a log-normal random variable with zero mean and standard deviation of $8 d B$ [7]. Therefore, the shadowing fading margin $X_{\sigma}$ is set $8 \mathrm{~dB}$.

9. The STAs use ommidirectional antennas (i.e. gain of $0 \mathrm{dBi}$ ).

10. The received power as a function of the distance $d$ is given by

$$
P_{r X, i}(d)=E I R P-L(d)-X_{\sigma}+G_{r X} .
$$

11. The data rate depends upon the PHY layer (see Table 1).

12. The energy per bit in a linear scale is given by $P / R$, where $P$ denotes the power and $R_{b}$ the bit rate [13, p. 185]. So, the energy per bit in $d B$-Joules is given by

$E_{b}=P_{r x}-R$.

13. The one side noise spectral density $N_{0}$ models the additive white Gaussian noise (AWGN) at the receiver input. It is given by

$N_{0}=k \cdot T_{0}$,

where the Boltzaman constant $k$ is equals to $-198.60 \mathrm{dBm} / \mathrm{K}-\mathrm{Hz}$ and $T_{o}$ is the effective noise temperature in degrees Kelvin (k). Assuming, $T_{0}=270{ }^{\circ} \mathrm{K}(24.31 \mathrm{~dB} / \mathrm{K})$, then $N_{o}=-174 \mathrm{dBm} / \mathrm{Hz}$.

14. The bandwidth $W$ depends on the PHY layer: (a) $W=20 \mathrm{MHz}$ (73 $\mathrm{dB}-\mathrm{Hz}$ ) for 802.11a; (b) $\mathrm{W}=22 \mathrm{MHz}(73.4 \mathrm{~dB}-\mathrm{Hz}$ ) for 802.11g.

15. The noise figure $F$ is defined by the ratio of the SINR at the input of a network to the SNR at the output at the network. It measures the noise introduced by the front-end amplifier at the receiver. We have assumed a typical value of $5 d B$.

16. The noise power in $\mathrm{dBm}$ at the detector input is given by

$N=N_{0}+W+F$.

17. The interference margin counts for co-channel interference, non-linear intermodulation effects, etc. It is assumed a value of $3 \mathrm{~dB}[8]$.

18. The total interference-plus-noise in $d B m$ is given by

$I=N_{0}+W+F+M$,

and, consequently, the interference-plus-noise spectral density in $\mathrm{dB} / \mathrm{Hz}$ is given by

$I_{0}=N_{0}+F+M$. 
19. The received SINR in linear scale is generically calculated by (34) $[13$, p. 185]. Therefore, the energy per bit to one side noise spectral density received at the detector input in $\mathrm{dB}$ is given by (35). The equation (35), using (28), can be rewritten as (40).

$$
\begin{aligned}
& \operatorname{SINR}=\frac{E_{b}}{I_{0}}=\frac{P_{r x}}{I_{0}}\left(\frac{1}{R_{b}}\right) \\
& (\operatorname{SINR})_{r x}=P_{r x}-N_{0}-F-M-R_{b} \\
& (\operatorname{SINR})_{r x}=E I R P-L(d)-X_{\sigma}+G_{r x}-N_{0}-F-M-R_{b} .
\end{aligned}
$$

20. The lower bound for the received SINR can be set by the following means: (a) analytically; (b) simulation; (c) field measures. In this paper, we have used the analytical framework developed in Sections 2 to 4.

21. Our objective is to estimate the cell range $d$ given a system configuration and a performance target (that is determined by $\mathrm{SINR}_{\mathrm{rx}}$ ). Hence, from (36), we can determine the maximum path loss using (37). Finally, the maximum cell range $d$ can be estimated using (26).

$$
L(\mathrm{~d})=E I R P+G_{r X}-\left\{(\operatorname{SINR})_{r X}+X_{\sigma}+N_{0}+F+M+R_{b}\right\} .
$$

\section{ANALYTICAL AND SimULATION RESUlts}

The simulation results are obtained using a $\mathrm{C}$ object oriented IEEE 802.11 joint MAC and PHY simulator [4].

Tab. III shows the IEEE 802.11a and 802.11g PHY layer parameters. It is also assumed: propagation delay $a=1 \mu . s$, MAC data payloads of 1023 octets, Rayleigh flat fading uncorrelated fading channel.

The analytical expressions that have been used to estimate the packet success probability for temporally uncorrelated Rayleigh fading channels can be found in [4], where it was assumed hard decision Viterbi decoding.

Table IV shows the ACs parameters. Henceforth, due to space constraints, we shall only present a set of results that assume an equal number of AC 1 and AC 3 QTAS (i.e. ACs that use different values for the AIFS and CW parameters).

TABLE III

IEEE 802.11a and 802.11g PHY layer parameters.

\begin{tabular}{|c|c|c|}
\hline Parameters & $802.11 \mathrm{a}$ & $802.11 \mathrm{~g}$ \\
\hline Slot time $\sigma$ & $9 \mu \mathrm{S}$ & $20 \mu \mathrm{S}$ \\
\hline SIFS & $16 \mu \mathrm{s}$ & $10 \mu \mathrm{S}$ \\
& & $20 \mu \mathrm{s}$ \\
\hline Preamble Duration & $20 \mu \mathrm{s}$ & $4 \mu \mathrm{s}$ \\
\hline PLCP header duration & $4 \mu \mathrm{s}$ & \\
\hline
\end{tabular}

TABLE IV

The IEEE 802.11e access classes.

\begin{tabular}{|c|c|c|c|}
\hline$A C$ & AIFS & $C W \min$ & $C W_{\max }$ \\
\hline 1 & $34 \mu \mathrm{s}$ & 32 & 1024 \\
\hline 2 & $25 \mu \mathrm{s}$ & 32 & 1024 \\
\hline 3 & $25 \mu \mathrm{s}$ & 16 & 32 \\
\hline 4 & $25 \mu \mathrm{s}$ & 8 & 16 \\
\hline
\end{tabular}

We show in Fig. 2 the IEEE 802.11a goodput as a function of the average signal-to-interference-plus-noise ratio (SINR) per bit for a system without spatial diversity (i.e. $L=1)$. It is used the PHY layers that optimise the goodput using a link adaptation procedure similar to that one described in [4]. The PHY layers that optimise the system performance depend upon complex interrelations of a multitude of factors: modulation schemes, channel coding, channel characteristics (in the time, frequency and spatial domain), receiver structures, traffic load, payload length and so forth. Analysing Fig. 2, we can promptly verify: (1) a good agreement between analytical and simulation results; (2) the major bandwidth, as expected, is allocated to AC3 QSTAs due to the efficient EDCF prioritisation mechanism.

We plot in Fig. 3, using the same parameters employed at Fig. 2, the IEEE 802.11a cell coverage as a function of the goodput. The cell range is estimated using (37), where the values of $(S I N R)_{r x}$, and their connections with the goodput, are obtained from Fig. 2.

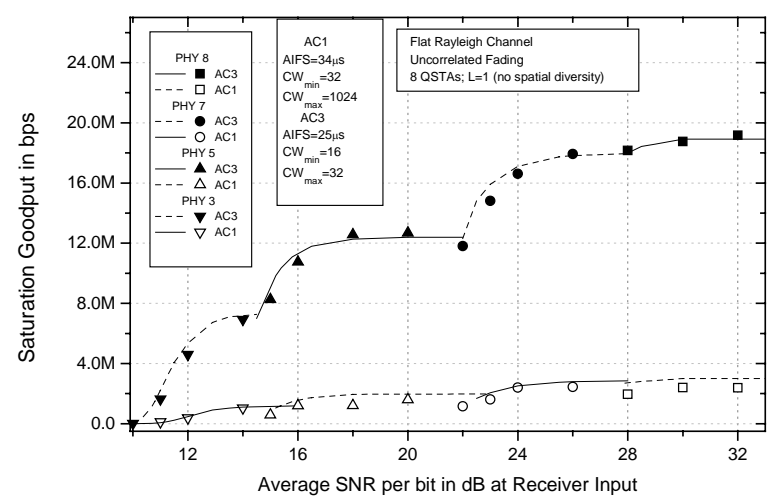

Fig. 2 Comparison between analytical (straight lines) and simulation (marks) results for the IEEE 802.11a goodput assuming four AC1 and four AC3 QSTAs.

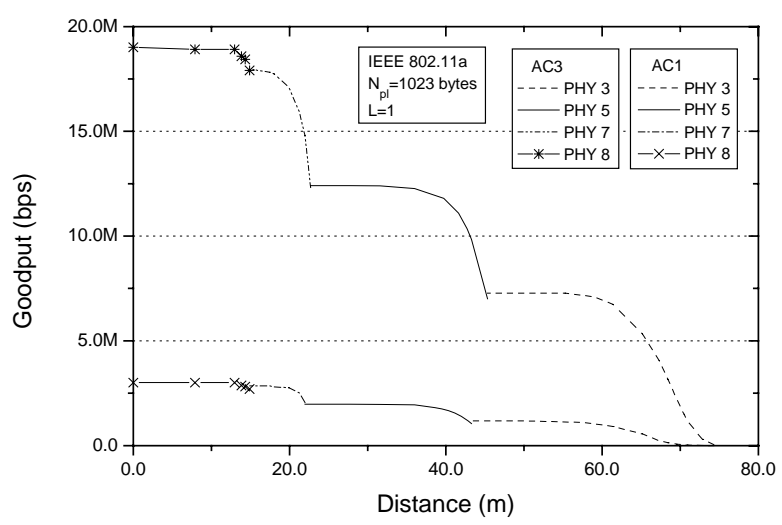

Figure 3. Cell coverage versus goodput for IEEE 802.11a.

$\mathrm{EIRP}=20 \mathrm{dBm}$. Channel $36(\mathrm{fc}=5.180 \mathrm{GHz}) . \mathrm{l}_{\mathrm{pl}}=1023$ bytes.

We show in Fig. 4 the IEEE 802.11g goodput as a function of the average SINR per bit for a system without spatial diversity, using an adaptive modulation scheme [4]. First, we notice a good agreement between analytical (straight lines) and simulation (marks) results. Second, comparing Fig. 4 with Fig. 2 we can see that the IEEE 802.11a provides a superior performance. This occurs because in the 802.11g ERP-OFDM: (1) the RTS/CTS control frames are transmitted using the 802.11b CCK modulation scheme to provide backward compatibility, as explained in Section IV. Notice that this modulation scheme is more sensible to the fading due the lack of error control coding; (2) there is a greater overhead due the SIFS, as shown in Tab. III.

Fig. 4 also shows that the better performance for IEEE 802.11 is obtained with PHY modes 7 and 8. These results are different from the ones showed for IEEE 802.11a at Fig. 2 (where the system is optimised with PHY modes 3, 5, 7 and 8). This use of different PHY modes occur because the 802.11g PHY layer is very sensible to errors that occur in the short length RTS and CTS control frames since they are transmitted without FEC coding. 
Fig. 5 depicts, using the same parameters employed at Fig. 4, the IEEE 802.11g cell coverage as a function of the goodput. The cell range is estimated using (37), where the values of $(S I N R)_{r x}$ are obtained from Fig. 4.

It is fundamental to remark that some white papers claim a superior range of $802.11 \mathrm{~b}$ in relation of 802.11a networks. This occurs because they have used the radio sensibility (which is the minimum signal level for the receiver to acceptably decode the information) to calculate the link budget. However, the radio sensibility does not consider the effects of fading and packet length on the system performance. To include these factors it is necessary to define a short-term fading margin in the link budget. This fading margin is not constant since it depends upon the environment, packet length and so forth. In our methodology, the fading margin is implicitly included in the calculation of the goodput since the transmission probability (see 7,9 and 10), the conditional collision probability (see 2 and 6), the cycle time (see 16-23) and the packet success probability (see [4) depends on the traffic load, payload length, SINR per bit and so forth.

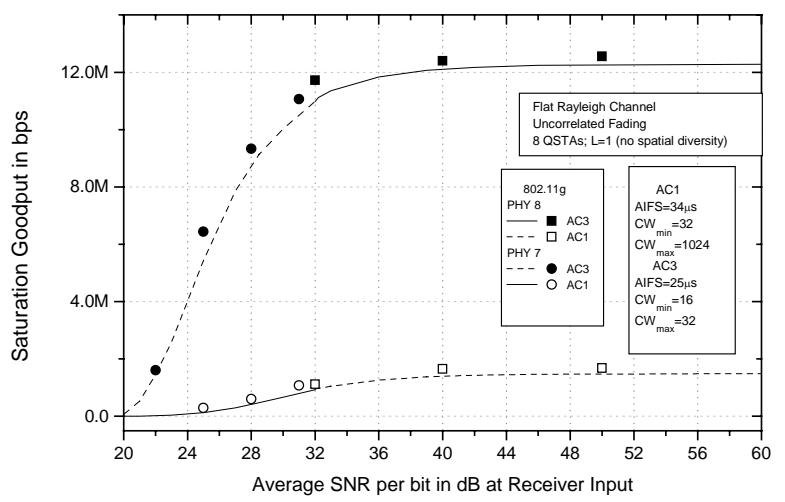

Fig. 4. Comparison between analytical (straight lines) and simulation (marks) results for the IEEE 802.11g goodput assuming four AC1 and four AC3 QSTAs.

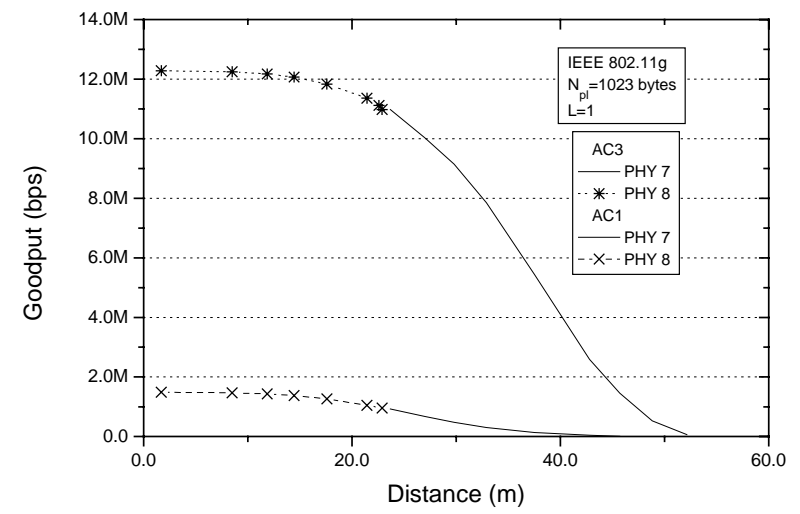

Fig. 5. Cell coverage versus goodput for IEEE 802.11g.

$\mathrm{EIRP}=24 \mathrm{dBm}$. Channel 36 (fc=2.412 GHz). $\mathrm{l}_{\mathrm{pl}}=1023$ bytes.

Finally, Fig. 6 shows the coverage of an IEEE 802.11a network hypothetically deployed in $2.5 \mathrm{GHz}$ Industrial, Scientific and Medical (ISM) band. Hypothetically because there is a necessity of backward compatibility with the large bases of 5.5 and 11 Mbps IEEE 802.11b operating in the $2.5 \mathrm{GHz}$ band as seen in Section 4. Comparing Fig. 6 with Fig. 3 we can see that this provides a substantial improvement due to the following reasons: (1) the lower path loss (see 26-27); (2) the maximum EIRP power in the ISM band is $24 \mathrm{dBm}$ and $20 \mathrm{dBm}$ in the $5 \mathrm{GHz}$ band.

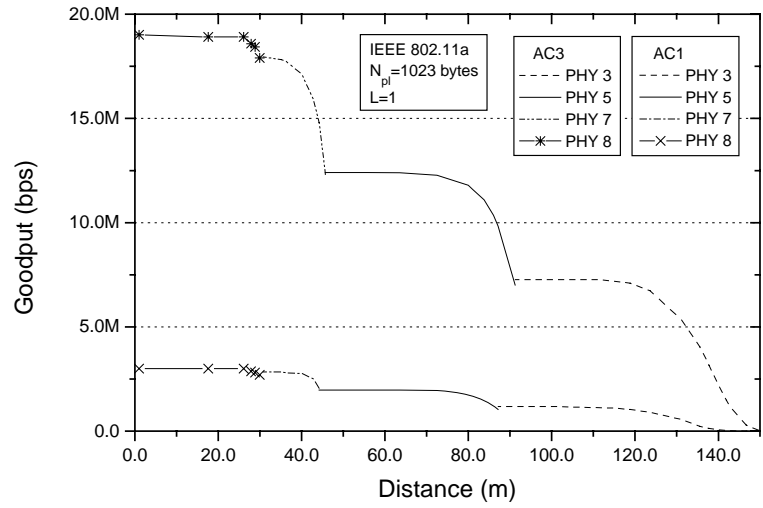

Figure 6. Cell coverage versus goodput for IEEE 802.11a operating hypotecally in the $2.4 \mathrm{GHz}$ ISM band.

EIRP=24 dBm. Channel $36\left(\mathrm{fc}_{\mathrm{c}}=2.412 \mathrm{GHz}\right) . \mathrm{l}_{\mathrm{pl}}=1023$ bytes.

\section{FINAL REMARKS}

The analytical, numerical and simulation results developed in our contribution allows to take into account in the channel load, goodput, channel modelling, receiver structures and link analyses for IEEE 802.11e networks in an integrated way. This contribution, to the best of our knowledge, helps to fill a gap in the open literature since it allows network designers to jointly analyse and optimize the performance and cell coverage of IEEE 802.11e networks. As future research directions, we have been developing analytical models for IEEE 802.11n (the new standard being developed that provides data rates in excess of $100 \mathrm{Mbps}$ and it is based on Multiple Input Multiple Output, MIMO, antenna architecture) [10].

\section{REFERENCES}

[1]B. P. Crow et al. "IEEE 802.11 wireless local area networks," IEEE Communications Magazine, vol. 35, no. 9, pp. 116-126, Sept. 1997.

[2]G. Bianchi "Performance Analysis of the IEEE 802.11 Distributed coordination function,” IEEE Journal on Select Areas on Comm., v.18, no. 3, pp. 535-547, March 2000.

[3]S. D. Qiao, S. Choi and K. G. Shin, "Goodput analyzes and link adaptation for the IEEE 802.11a wireless LANs,” IEEE Trans. Mobile Comp., pp. 278-292, 2002.

[4] R. P. F. Hoefel. “A MAC and PHY cross-layer analytical model for the goodput and delay of IEEE 802.11a networks operating under Basic Access and RTS/CTS DCF schemes", Journal of Communications (JCM), v.1, n.6, p. 1-8, Set. 2006.

[5]J. W. Robinson and T. Randhawa, "Saturation throughput analysis of IEEE 802.11e enhanced distributed coordination function", IEEE Journal on Select. Areas on Comm., vol. 22, no. 5, p. 917-928, June 2004.

[6]R. P. F. Hoefel. “An Analytical Cross-Layer Model for the Goodput and Delay of IEEE 802.11e Enhanced Distribution Coordination Function”, in Proc. of $64^{\text {th }}$ IEEE Vehicular Technology 2006 Fall (VTC2006-Fall), 2006.

[7] M. V. Clark et. Al. “Outdoor IEEE 802.11 Cellular Networks: Radio Link Performance,” in Proc. of IEEE ICC, 2002.

[8] I. Ramela and J. F. de Rezende. “Avoiding primary interference with directional antennas in Ad Hoc networks” (in Portuguese), in Proc. of $24^{\text {th }}$ Brazilian Symposium of Computer Networks, 2006.

[9] R. P. F. Hoefel. "A MAC and PHY Cross-Layer Model to Estimate the Cell Coverage of IEEE 802.11a, 802.11b and 802.11g WLANs,” in Proc. of $25^{\text {th }}$ Brazilian Symposium of Computer Networks, 2007.

[10] M. S. Gast, 802.11 Wireless Networks. New York: O’Reilly, 2005.

[11] R. P. F. Hoefel. “A Cross-Layer Model for HR/DSSS IEEE 802.11b WLANs with Differentiation Services” in Proc. 2006 IEEE Ninth Inte. Symp.Spread Spectrum Techniques and Applications, 2006.

[12] Hashemi, H. (1993) "The indoor radio propagation channel", Proceedings of IEE, v. 81, n. 7, p. 943-968, 1993

[13] B. Skalar. “Digital Communications”, Upper Saddle River: Pearson Education, $3^{\text {th }}$ edition. 